\title{
Atypical syndromes caudal to the injury site in patients following spinal cord injury. A clinical, neurophysiological and MRI study
}

\author{
L Tosi MD, ${ }^{1} \mathrm{C}$ Righetti MD,${ }^{1} \mathrm{G}$ Terrini MD,${ }^{1} \mathrm{G}$ Zanette $\mathrm{MD}^{2}$ \\ ${ }^{1}$ Department of Rehabilitation, Neurophysiological Laboratory, Don Calabria Hospital, \\ 37024 Negrar, Verona, Italy; ${ }^{2}$ Institute of Neurology, University School of Medicine, \\ Verona, Italy.
}

\begin{abstract}
Some patients with a cervical or a high thoracic spinal cord injury show atypical signs and symtoms such as atrophy of lower leg muscles, reduced tendon jerks or urinary retention, either persisting from the acute phase or developing in the course of time. A series of 35 patients was prospectively examined from this point of view with neurophysiological, urodynamic and MRI studies. Seven patients $(20 \%)$ presented atypical findings; in two there was an isolated areflexic bladder, but no signs of functional or structural sacral damage were found. In five patients $(14.2 \%)$ a definite cause for the sacral lesion could be detected. The syndrome caudal to the level of the lesion is neither obvious at the beginning nor immutable in time. Treatment requires to be adapted to the changing patterns of somatic and visceral activity.
\end{abstract}

Keywords: spinal cord injury; atypical clinical syndromes; neurogenic bladder dysfunction; posttraumatic syringomyelia; lumbosacral evoked potentials; magnetic resonance imaging.

\section{Introduction}

We traditionally understand that patients with a cervical or a thoracic spinal cord injury (SCI), once the stage of spinal shock has subsided, typically develop signs of upper motor neuron dysfunction below the level of the lesion: increased muscle tone, exaggerated tendon jerks, clonus and a hyperreflexic bladder. ${ }^{1}$ Some years ago we observed a patient with traumatic SCI at T5 level, with atypical findings such as atrophy of leg muscles, reduced tendon jerks and urinary retention, 2 years postinjury. In 1987 a paper by Beric and coworkers focused attention on such atypical cases, with clinical, neurophysiological and urodynamic evidence for an "occult" sacral lesion. ${ }^{2}$ Since then we have carefully examined patients with SCI in this light and have performed a systematic investigation to detect any possible sacral damage. A distinction between typical and atypical cases was sought at every step of the investigation. The hope was that MRI, a powerful tool in the follow up management of $\mathrm{SCI},{ }^{3}$ could now detect previously occult causes of a sacral lesion.

\section{Patients and methods}

Thirty-five patients with long-standing traumatic SCI, consecutively observed from September 1987 to December 1991, are included in this prospective study. The sample consists of 27 males and eight females, aged from 18 to 60 years (mean age: 35 years). They had sustained a traumatic SCI within a minimum of 1 year and a maximum of 20 years prior to the evaluation. The level of the lesion ranged from neural segments C3 to T8. Three patients with obvious causes of lumbosacral damage (fractures, bullet wounds) were excluded.

With conventional neurological examination particular attention was paid to evidence of diminished spasticity, reduced or absent tendon jerks, atrophy of lower leg muscles and urinary retention, as well as to the presence or absence of anal skin reflexes.

Neurophysiological examination consisted of the following:

1 Electromyography with concentric needles in the quadriceps, tibialis anterior and gastrocnemius muscles, to determine any 
denervation and assess any voluntary motor unit recruitment.

$2 \mathrm{~F}$ waves from the extensor brevis digitorum and flexor hallucis muscles in response to supramaximal $1 \mathrm{~Hz}$ stimulation of the peroneal and tibial nerves at the ankle, 40 consecutive stimulations being applied to each nerve. Lack of response or minimal latency above $54 \mathrm{~ms}$ for the peroneal and $56 \mathrm{~ms}$ for the tibial nerve were regarded as pathological.

$3 \mathrm{H}$ reflexes, stimulating the tibial nerve at the knee with $0.2 \mathrm{~Hz}$ and graded intensity to obtain maximal $\mathrm{H}$ and $\mathrm{M}$ response, recording from the triceps surae muscle. Lack of response or latency of more than $43 \mathrm{~ms}$ were regarded as pathological.

4 Bulbocavernosus reflex, stimulating the dorsal nerve of the penis with ring electrodes or the dorsal nerve of the clitoris with clip-type electrodes, recording from the perineal muscles. Lack of response or latency of above $40 \mathrm{~ms}$ were regarded as pathological.

5 Lumbosacral somatosensory evoked potentials (LSEPs), stimulating the tibial nerve at the ankle with square-wave stimuli of $0.1 \mathrm{~ms}$ duration and $3 \mathrm{~Hz}$ frequency and an intensity corresponding to twice the motor threshold. In patients with denervation, an intensity sufficient to evoke a maximal sensory potential of the sciatic nerve was chosen. Monopolar needle electrodes were applied over the gluteal fold (GF, bipolar recording), the spinous processes with common reference to the ipsilateral iliac crest and the $\mathrm{Cz}$ point with frontal reference. The ampli- fier bandwidth was from 5 to $1000 \mathrm{~Hz}$ for the scalp and from 20 to $1000 \mathrm{~Hz}$ for the other leads. Analysis of the tracings, extrapolated by averaging 700 artefactfree responses, was done over the first $100 \mathrm{~ms}$. LSEPs were carried out in 21 out of 28 typical cases and in all seven atypical cases. Amplitude of GF, N18 ('R' wave) at S1 and N22 ('S' wave) at T12, as well as latency of GF, interpeaks GF-N18 and GF-N22 were determined. Twenty age matched volunteers served as controls. Reference data are included in Table I.

In urodynamic testing a constant inflow rate of $60 \mathrm{ml} / \mathrm{min}$ was used to fill the bladder with an infusion pump connected up to a double-lumen catheter. The other lumen was attached to a transducer to monitor the intravesical pressure. The results were recorded and processed on DISA 2100. Abnormal bladder behaviour was categorised as follows: (1) hyperreflexic with good emptying (residual urine less than $100 \mathrm{ml}$ ); (2) hyperreflexic with poor emptying due to detrusor sphincter dyssinergia (DSD): (3) hyperreflexic with poor emptying due to unsustained detrusor contraction; and (4) areflexic. When the overall body of data thus obtained suggested sacral damage, a targeted regional MRI study was carried out.

\section{Results}

From the 35 patients studied, 28 (15 with a complete and 13 with an incomplete spinal

Table I Lumbosacral somatosensory evoked potentials (LSEPs)

\begin{tabular}{|c|c|c|c|c|c|c|}
\hline & \multirow{2}{*}{\multicolumn{2}{|c|}{$\begin{array}{l}\text { Control } \\
\text { group } \\
(n=20)\end{array}$}} & \multicolumn{4}{|c|}{ Spinal lesions ${ }^{\mathrm{a}}$} \\
\hline & & & \multicolumn{2}{|c|}{$\begin{array}{l}\text { Partial } \\
(n=12)\end{array}$} & \multicolumn{2}{|c|}{$\begin{array}{l}\text { Complete } \\
(n=11)\end{array}$} \\
\hline & Mean & SD & Mean & SD & Mean & SD \\
\hline GF-N18 (ms) & 5.2 & 0.6 & 5.4 & 0.7 & 5.2 & 0.5 \\
\hline GF-N22 (ms) & 8.5 & 0.6 & 8.3 & 0.8 & 8.5 & 0.5 \\
\hline $\mathrm{N} 18(\mathrm{uV})$ & 1.6 & 0.8 & 1.5 & 0.6 & 1.7 & 0.9 \\
\hline N22 (uV) & 0.8 & 0.3 & 0.7 & 0.4 & 0.8 & 0.5 \\
\hline
\end{tabular}

aTwo cases with isolated bladder areflexia are included. Five cases with sacral lesion and absent LSEPs are excluded. There are no significant differences between normal and SCI groups. 
lesion) presented a typical syndrome below the level of the lesion with spasticity, normal somatic reflex responses, normal LSEPs (21 patients) and a hyperreflexic bladder with or without DSD. Anal skin reflexes were present in all of these patients.

Two patients presented an areflexic bladder, in one case 18 and in the other 23 months after trauma, with an otherwise typical syndrome with spasticity, normal somatic reflex activity and normal LSEPs. Anal skin reflexes were also present. Sacral MRI was normal.

Five patients presented with atypical clinical signs: abnormal somatic reflex activity, abnormal LSEPs and areflexic bladder, and absent anal skin reflexes. In this group of atypical cases, two patients with a lesion level at T6 and T8 still exhibited flaccid paraplegia with lack of tendon reflexes (knee and ankle) and urinary retention 1 year after trauma. Spontaneous denervation activity was present on EMG, segmental reflex responses and LSEPs were absent and the bladder was areflexic. MRI provided evidence of spinal cord atrophy below the level of injury, extending as far as the conus medullaris (Fig 1). Another two atypical patients, with lesions at C5 and T2, presented initially with a typical syndrome, including spasticity and a hyperreflexic bladder. Three and 10 years, respectively, after trauma the clinical picture slowly changed, with reduction of spasticity, disappearance of ankle reflexes, distal muscular atrophy and bladder areflexia. At the time of examination, somatic reflex activity and LSEPs were abnormal. MRI revealed multiple cystic cavities or small syrinxes in the spinal cord, extending from the injury site to the sacral region, where they were even larger (Fig 2). The fifth atypical patient had spasticity and a hyperreflexic bladder for 20 years, changing to flaccidity, atrophy, areflexia and urinary retention within a few weeks of a herpes zoster infection, 2 years prior to our examination. At EMG neither spontaneous nor voluntary activity were present. No conduction velocity study was obtainable. MRI showed a cystic cavity at the level of lesion but no abnormality of the sacral cord. The findings are summarised in Table II.

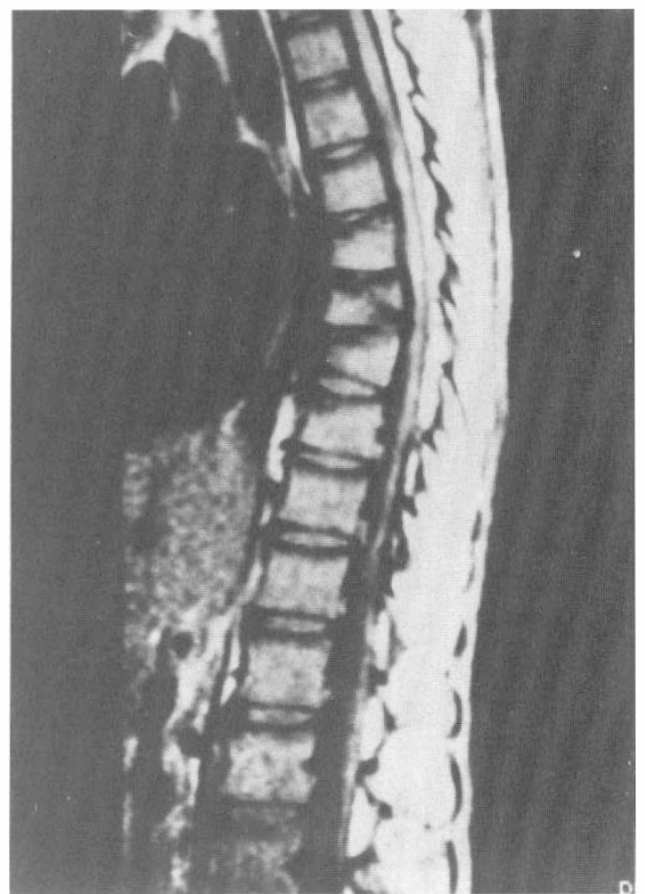

Figure 1 MRI of a patient with a spinal cord injury at T8, showing atrophy of the cord below the injury site.

\section{Discussion}

Traumatic spinal cord injury usually causes a localised lesion with limited extension to adjacent areas. Once the spinal shock phase has passed, a stable clinical syndrome of spasticity and bladder hyperreflexia sets in, these phenomena being regarded as an expression of a 'release' of spinal reflex activity. The anal skin reflexes reappear first, possibly not having disappeared at all. ${ }^{4}$ Neurophysiological studies in patients with a SCI show no change in spinal potentials caudal to the lesion, ${ }^{5}$ while cortical SEPs show varying degrees of abnormality. ${ }^{6}$ This was the picture observed in our typical cases $(80 \%)$.

In two patients the only unexpected finding was detrusor areflexia, 18 and 23 months after trauma, in an otherwise typical syndrome with spasticity and normal neurophysiological findings. The regional sacral MRI was normal. These observations are in 


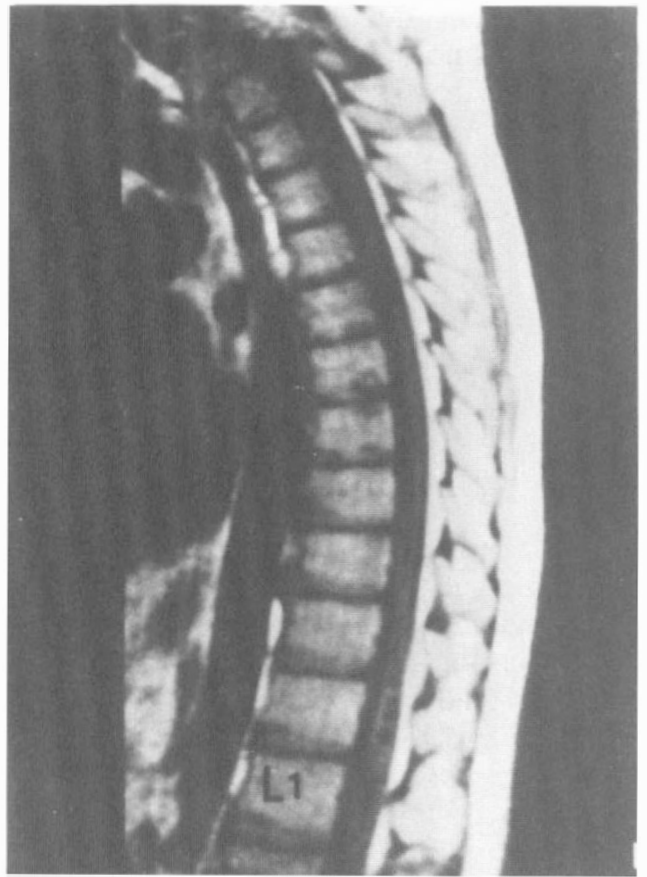

Figure 2 MRI of a patient with a spinal cord injury at $\mathrm{T} 2$, showing multiple cysts and small syrinxes, these being larger in the sacral region.

agreement with data reported by Light et al, ${ }^{7}$ Lucas and Thomas ${ }^{8}$ and Kaplan et $a l^{4}$ : isolated persistent or permanent bladder areflexia, by no means a rare occurrence with high spinal cord lesions, does not imply either impairment of sacral somatic reflex activity or a demonstrable sacral lesion. Bladder activity may return much later than the somatic reflex activity. ${ }^{7}$ No correlation has been confirmed between neurophysiological parameters and patterns of bladder and sphincter behaviour following SCI, provided that there is no definite sacral lesion. ${ }^{8}$ Five patients $(14.2 \%)$ presented clinical signs of sacral dysfunction (reduced or absent tendon jerks, distal muscular atrophy, persistent urinary retention), associated with neurophysiological abnormalities (denervation, abnormal somatic reflex activity, abnormal LSEPs) and an areflexic bladder. Beric et $a l^{2}$ in 130 patients with cervical and thoracic injuries, selected an analogous group of 15 cases $(11.5 \%)$ who presented with unexpected signs of a sacral lesion. Acute traumatic or vascular lesion,

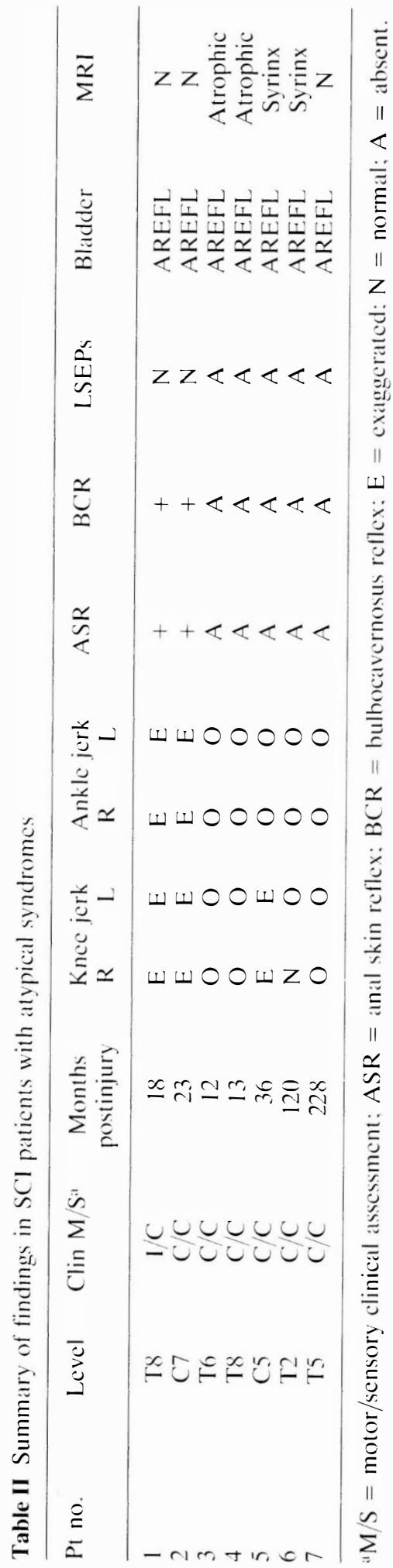


posttraumatic syringomyelia or arachnoiditis, spinal stenosis or tethering of the cord were assumed to be hypothetical causes. In four of our patients, MRI revealed a clearly defined sacral lesion.

In two cases, the neurological syndrome was atypical, in that they never developed spasticity and automatic bladder emptying. Failure of reflex return will occur if the transverse lesion is associated with a lesion in the vertical plane. ${ }^{1}$ Both patients were polytraumatized, with major chest lesions and traumatic shock in the acute phase. At MRI the whole cord distal to the injury had become atrophic. This structural damage is probably due to a secondary acute spinal cord injury with vascular mechanism, ${ }^{10}$ since systemic hypotension, local vasospasm and loss of vascular self-regulation are unquestionably major factors in aggravating and extending spinal cord damage.

In two other cases, MRI detected multiple cystic cavities or syrinxes. Posttraumatic cystic degeneration of the cord or syringomyelia were thought to be rare sequelae of SCI, occurring in less than $3.2 \%$ of the patients." Recent MRI studies reveal a much higher prevalence of cystic evolution in SCI patients. ${ }^{12.13}$ The clinical presentation is marked by the appearance of new symptoms developing after neurological stabilisation. Increasing myelopathy with alteration in the Frankel grade, an ascending neurological level, pain, hyperhydrosis or increasing muscular spasm of recent onset are the features of the clinical presentation. ${ }^{13}$ The change from a typical to an atypical syndrome below the level of the lesion can be added to these patterns. MRI is now a simple noninvasive method of diagnosing syringomyelia. Pathogenesis and treatment remain open questions. ${ }^{14.15}$

In the fifth case, the change from a typical to an atypical syndrome was impressive. The patient, who had a lesion at T5 in 1968, had severe disturbing spasticity and an efficient reflexic bladder for 20 years. In a few weeks of a herpes zoster infection, the situation changed to one of areflexia, atrophy of leg muscles and urinary retention. There was no change in the arms and trunk. MRI showed no abnormality of the sacral cord. A herpertic myeloradiculitis can be considered as a possible cause. It is interesting that this now 'atypical SCI patient' lives a better life than he did previously.

Based on our experience with these patients, we summarise the main features for the recognition of atypical syndromes. The leading clinical findings are absent or decreasing spasticity, absence or regression of the anal skin reflexes, and incongrouous bladder behaviour. The leading investigation is MRI of the sacral region. Recognition of an atypical syndrome caudal to the initial injury level has some implications for the patient's management. With reference to the final state, treatment of a spastic paraplegic patient with a hyperreflexic bladder is obviously different from the treatment of a patient with a flaccid paraplegia and an areflexic bladder. Problems arise in the transitional phases, when physical therapy and bladder management must be adapted to the changing patterns of somatic and visceral activity. A particular difficulty may arise when a prior hypereflexic, efficiently emptying bladder changes into one with poor emptying due to unsustained detrusor contraction (cases 5, 6, 7). Sphincterotomy in these cases failed to improve bladder emptying, owing to decreasing detrusor contractility. According to Light et al, ${ }^{16}$ sphincterotomy in patients with a high spinal cord injury and signs of sacral damage is of no use, since detrusor contractility may be adversely affected.

\section{Conclusions}

1 In patients with a cervical or a thoracic SCI, the syndrome caudal to the level of the lesion is neither obvious from the beginning nor immutable in time: it is atypical in an appreciable number of cases (15-20\%).

2 Atypical clinical signs and abnormal somatic reflex responses suggest unexpected or occult damage.

3 MRI can detect the otherwise occult damage in most cases.

4 An isolated areflexic bladder does not imply detectable sacral damage.

5 Clinical and instrumental monitoring may identify atypical courses, calling for an appropriate therapeutic approach. 


\section{References}

1 Guttmann L (1976) Patterns of reflex disturbances. In: Spinal Cord Injuries. 2nd ed. Blackwell Scientific Publications, Oxford: 241.

2 Beric A, Dimitrijevic MR, Light JK (1987) A clinical syndrome of rostral and caudal spinal injury: neurological, neurophysiological and urodynamic evidence for occult sacral lesion. $J$ Neurol Neurosurg Psychiatry 50: 600-606.

3 Sett P, Crockard HA (1991) The value of magnetic resonance imaging (MRI) in the follow-up management of spinal injury. Paraplegia 29: 396-410.

4 Lucas MG, Thomas DG (1989) Lack of relationship of conus reflexes to bladder function after spinal cord injury. Br J Urol 63: 24-27.

5 Sedgwick EM, El-Negamy E, Frankel H (1980) Spinal cord potentials in traumatic paraplegia and quadriplegia. J Neurol Neurosurg Psychiatry 43: 823-830.

6 Beric A (1992) Cortical somatosensory evoked potentials in spinal cord injury patients. J Neurol Sci 107: 50-59.

7 Light JK, Faganel J, Beric A (1985) Detrusor areflexia in suprasacral spinal cord injuries. J Urol 134: $295-297$.

8 Lucas MG, Thomas DG (1990) Lumbosacral evoked potentials and vesicouretral function in patients with chronic suprasacral spinal cord injury. J Neurol Neurosurg Psychiatry 53: 982-986.

9 Kaplan SA, Chancellor MB, Blaivas JG (1991) Bladder and sphincter behaviour in patients with spinal cord lesions. J Urol 146: 113-117.

10 Tator CA, Fehlings MG (1991) Review of the secondary injury theory of acute spinal cord trauma with emphasis on vascular mechanism. J Neurosurg 75: 15-26.

11 Rossier AB, Foo D, Shillito J, Dyro EM (1985) Post-traumatic cervical syringomyelia. Incidence. clinical presentation, electrophysiological studies, syrinx protein and results of conservative and operative treatment. Brain 108: 439-461.

12 Backe HA, Betz RR, Mesgarzadeh M (1991) Post-traumatic cord cysts evaluated by magnetic resonance imaging. Paraplegia 29: 607-612.

13 Silberstein M, Hennessy O (1992) Cystic cord lesions and neurological deterioration in spinal cord injury: operative considerations based on magnetic resonance imaging. Paraplegia 30: 661-668.

14 Williams B (1990) Post-traumatic syringomyelia, an update. Paraplegia 28: 296-313.

15 Umbach J, Heilporn A (1991) Review article: postspinal cord injury syringomyelia. Paraplegia 29: $219-221$.

16 Light JK, Beric A, Wise PG (1987) Predictive criteria for failed sphincterotomy in spinal cord injury patients. J Urol 138: 1201-1204. 